\title{
Long path lemma concerning connectivity and independence number
}

\author{
Shinya Fujita* Alexander Halperin $^{\dagger} \quad$ Colton Magnant $^{\ddagger}$ \\ Submitted: May 10, 2010; Accepted: Nov 26, 2011; Published: Jul 22, 2011 \\ Mathematics Subject Classification: 05C35
}

\begin{abstract}
We show that, in a $k$-connected graph $G$ of order $n$ with $\alpha(G)=\alpha$, between any pair of vertices, there exists a path $P$ joining them with $|P| \geq \min \left\{n, \frac{(k-1)(n-k)}{\alpha}+k\right\}$. This implies that, for any edge $e \in E(G)$, there is a cycle containing $e$ of length at least $\min \left\{n, \frac{(k-1)(n-k)}{\alpha}+k\right\}$. Moreover, we generalize our result as follows: for any choice $S$ of $s \leq k$ vertices in $G$, there exists a tree $T$ whose set of leaves is $S$ with $|T| \geq \min \left\{n, \frac{(k-s+1)(n-k)}{\alpha}+k\right\}$.
\end{abstract}

\section{Introduction}

In this work, we present a tool which we believe will be useful in many applications. Much work has been devoted to finding long paths and cycles in graphs. In particular, in [4], O, West and Wu recently proved a conjecture by Fouquet and Jolivet [3] stated as follows.

Theorem 1 ([4]) Let $k \geq 2$ and let $G$ be a $k$-connected graph of order $n$ with $\alpha(G)=\alpha$. Then there is a cycle in $G$ of length at least $\min \left\{n, \frac{k(n+\alpha-k)}{\alpha}\right\}$.

In various situations including this work, it often becomes necessary to find a long path between a chosen pair of vertices. For this reason, O, West and $\mathrm{Wu}$ proved the following theorem which they used in their proof of the conjecture.

Theorem 2 ([4]) Let $G$ be a k-connected graph for $k \geq 1$. If $H \subseteq G$ and $u$ and $v$ are distinct vertices in $G$, then $G$ contains a u,v-path $P$ such that $V(H) \subseteq V(P)$ or $\alpha(H-P) \leq \alpha(H)-(k-1)$.

*Department of Mathematics, Gunma National College of Technology. 580 Toriba, Maebashi, Gunma, Japan 371-8530. Supported by JSPS Grant No. 20740068

${ }^{\dagger}$ Department of Mathematics, Lehigh University, Bethlehem, PA 18015 USA

${ }_{\ddagger}^{\ddagger}$ Department of Mathematical Sciences, Georgia Southern University, Statesboro, GA 30460 USA 
We also use this theorem and, following the proofs presented in [4], we prove the following lemma which is our main result.

Lemma 1 Let $k \geq 1$ be an integer and let $G$ be a graph of order $n$ with $\kappa(G)=k$ and $\alpha(G)=\alpha$. Then for any pair of vertices $u, v$ in $G$, there exists a $u, v$-path of order at least $\min \left\{n, \frac{(k-1)(n-k)}{\alpha}+k\right\}$.

Our hope is that this lemma may be applied to produce other results like Theorem 3, which follows immediately from Lemma 1 by choosing $u$ and $v$ to be the ends of $e$.

Theorem 3 Let $k \geq 2$ be an integer and let $G$ be a $k$-connected graph of order $n$ with $\alpha(G)=\alpha$. Then for any edge $e \in E(G)$, there exists a cycle of length at least $\min \left\{n, \frac{(k-1)(n-k)}{\alpha}+k\right\}$ in $G$ containing the edge $e$.

Lemma 1 can be generalized to the following result concerning large trees with specified sets of leaves. Let $\ell(T)$ denote the set of leaves in a tree $T$.

Theorem 4 Let $k$ and $s$ be integers with $2 \leq s \leq k$ and let $G$ be a $k$-connected graph of order $n$ with $\alpha(G)=\alpha$. Then for any set of $s$ vertices $V_{s}=\left\{v_{1}, \ldots, v_{s}\right\} \subseteq G$, there exists a tree $T \subseteq G$ with $V_{s}=\ell(T)$ and $|T| \geq \min \left\{n, \frac{(k-s+1)(n-k)}{\alpha}+k\right\}$.

The proofs of Lemma 1 and Theorem 4 are presented in Section 3. As we will observe in Section 4, our results are all best possible.

\section{Preliminaries}

In our proof, we use the following corollary to break the problem into cases. We also state and prove a path version of Theorem 6. Both of these results come from [4].

Corollary 5 ([4]) If a graph $G$ admits no vertex partition $\left(V_{1}, V_{2}\right)$ such that $\alpha(G)=$ $\alpha\left(G\left[V_{1}\right]\right)+\alpha\left(G\left[V_{2}\right]\right)$, then $G$ is 2-connected or $G \in\left\{K_{1}, K_{2}\right\}$. Also, for distinct vertices $u, v \in G$, there is a $u, v$-path $P$ such that $\alpha(G-P)<\alpha(G)$.

Theorem 6 ([4]) Let $k$ be an integer greater than 1. If $C$ is a cycle with size at least $k$ in a $k$-connected graph $G$, then for any non-empty subgraph $H \subseteq G-C$, there exists a cycle $C^{\prime}$ in $G$ such that $\left|C-C^{\prime}\right| \leq \frac{|C|}{k}-1$ and $\alpha\left(H-C^{\prime}\right) \leq \alpha(H)-1$.

We will also make use of the following classical result of Chvátal and Erdös [2]. A graph is said to be hamiltonian connected if, between any pair of vertices, there exists a path covering the entire graph.

Theorem 7 ([2]) For any graph $G$, if $\kappa(G)>\alpha(G)$, then $G$ is hamiltonian connected.

Following the notation of [4], let $P$ be a path and $u$ and $v$ be vertices in $P$. Define $P(u, v)$ to be the subpath of $P$ strictly between (not including) $u$ and $v$. Also, for a vertex $v$ and a set of vertices or subgraph $A$, define a $(v, A) k$-fan to be a set of $k$ paths from $v$ to $A$ which are all pairwise vertex disjoint except at $v$. All other standard notation comes from [1]. 


\section{Proofs of our Main Results}

We begin by proving a key lemma used to obtain our main result. The main idea of the proof is based on that of Theorem 6 .

Lemma 2 Let $k \geq 2$ be an integer, and suppose $G$ is a $k$-connected graph containing vertices $u, v$. If $P$ is a $u, v$-path of order at least $k$ in $G$, then for any non-empty subgraph $H \subseteq G \backslash P$, there is a $u, v$-path $P^{\prime}$ in $G$ such that $\left|P \backslash P^{\prime}\right| \leq \frac{|P|-k}{k-1}$ and $\alpha\left(H \backslash P^{\prime}\right) \leq \alpha(H)-1$.

Proof: Suppose there exists a subgraph $H$ for which there is no desired path $P^{\prime}$ and choose $H$ to be the smallest such subgraph. By Corollary 5, either

(1) $H$ can be bipartitioned into non-empty subgraphs $H_{1}$ and $H_{2}$ so that $\alpha(H)=\alpha\left(H_{1}\right)+$ $\alpha\left(H_{2}\right)$, or

(2) $H$ is 2-connected or $H \in\left\{K_{1}, K_{2}\right\}$. Also, for any distinct vertices $x, y \in H$, there exists an $x, y$-path $P_{x y}$ in $H$ such that $\alpha\left(H \backslash P_{x y}\right)<\alpha(H)$.

If (1) holds, we simply apply Lemma 2 on $H_{1}$ (since $H$ was the smallest counterexample) and obtain a path $P^{\prime}$ satisfying the desired conditions. Hence we may assume (2) holds.

Let $B$ be the block of $G \backslash P$ containing $H$. First we assume $|B| \geq k$. By Menger's Theorem, there exist $k$ vertex-disjoint paths from $P$ to $B$. Choose the shortest such set of paths, meaning that each path contains exactly one vertex of $B$ and one vertex of $P$. This means that there must exist a pair of these paths, say $P_{1}=p_{1} \ldots b_{1}$ and $P_{2}=p_{2} \ldots b_{2}$ for $p_{i} \in V(P)$ and $b_{i} \in V(B)$ such that there are at most $\frac{|P|-k}{k-1}$ vertices between $p_{1}$ and $p_{2}$ on $P$. Since $B$ is 2-connected, there exist vertex-disjoint paths $P_{b_{i}}$ in $B$ from $b_{i}$ to $h_{i} \in V(H)$ for $i=1,2$. Note that $h_{1}=h_{2}$ is only possible if $|H|=1$. (Suppose $P_{b_{i}} \cap H=h_{i}$.) By (2), there is a path $P_{H}$ in $H$ from $h_{1}$ to $h_{2}$ for which $\alpha\left(H \backslash P_{H}\right)<\alpha(H)$. Then $P^{\prime}=\left(P \backslash P\left(p_{1}, p_{2}\right)\right) \cup\left(P_{1} \cup P_{b_{1}} \cup P_{H} \cup P_{b_{2}} \cup P_{2}\right)$ is the desired path. Hence, we may assume $|B|<k$.

Let $V(B)=\left\{b_{1}, \ldots, b_{\ell}\right\}$, where we have assumed $\ell<k$. Note that we may possibly have $\ell=1$. Let $C$ be the component of $G \backslash P$ containing $B$. Let $S=\left\{p_{1}, \ldots, p_{m}\right\}$ be the set of vertices of $P$ (in order along $P$ ) with at least one neighbor in $C$. Note that, by Menger's Theorem, $m \geq k$.

For each edge $e$ from $p_{i}$ to $C$, there exists a unique vertex $b_{j} \in B$ such that there is a unique path $Q_{i, j}$ from $b_{j}$ to $p_{i}$ containing $e$ with all interior vertices in $C \backslash B$. Let $X_{j}$ be the set of vertices $p_{i}$ for which such a path $Q_{i, j}$ exists. Note that the sets $\left\{X_{j}\right\}$ are not necessarily disjoint. Also note that, since $B$ is a block, $Q_{i, j}$ and $Q_{i^{\prime}, j^{\prime}}$ are internally disjoint when $j \neq j^{\prime}$. Call a segment $P\left(p_{i}, p_{j}\right)$ for $i<j$ large if $p_{i} \in X_{i^{\prime}}$ and $p_{j} \in X_{j^{\prime}}$ for some $i^{\prime} \neq j^{\prime}$. Otherwise, as long as the segment $P\left(p_{i}, p_{j}\right)$ is not contained in a large segment, it will be called small.

Using the same argument as above, the following fact is immediate.

Fact 1 For any large segment $P\left(p_{i}, p_{j}\right)$, we have 


$$
\left|P\left(p_{i}, p_{j}\right)\right|>\frac{|P|-k}{k-1} .
$$

Let $t$ be the number of segments $P\left(p_{i}, p_{i+1}\right)$ for $1 \leq i \leq m$ which are large. Since large segments contain at least $\frac{|P|-k+1}{k-1}$ vertices, we see that

$$
|P| \geq t\left(\frac{|P|-k+1}{k-1}\right)+k,
$$

which implies that $t<k-1$. For each $b_{i} \in B$, there exists a $\left(b_{i}-P\right) k$-fan. Choose such a fan so that each path intersects $P$ in exactly one vertex. Let $v_{1}, \ldots, v_{k}$ (in this order on $P$ ) be the vertices of $P$ at the ends of this fan. For each pair $v_{j}, v_{j+1}$, we already know that $v_{j}, v_{j+1} \in X_{i}$, but if one of these is also in $X_{i^{\prime}}$ for some $i^{\prime} \neq i$, then $P\left(v_{j}, v_{j+1}\right)$ must be a large segment of $P$. This means that, for each vertex in $B$, there are at least $k-1-t$ corresponding small segments of $P$. Since the ends of these small segments corresponding to $b_{i}$ are all in $X_{i}$, these segments must then be disjoint from all small segments corresponding to $b_{j}$ for $j \neq i$ since the ends of those segments would be in $X_{j}$. Therefore there are $(k-1-t) \ell$ small segments all pairwise disjoint. This implies that the average order of small segments is at most

$$
\frac{|P|-t\left(\frac{|P|-k+1}{k-1}\right)-k}{(k-1-t) \ell} .
$$

By the pigeonhole principle, if we choose the shortest small segment corresponding to each vertex $b_{i} \in B$, then the sum of the orders of these shortest segments is at most

$$
\frac{|P|-t\left(\frac{|P|-k+1}{k-1}\right)-k}{(k-1-t)} \leq \frac{|P|-k}{k-1} .
$$

We now replace each of these small segments with the corresponding $b_{i}$ using the paths $Q_{i, j}$ and $Q_{i, j+1}$ for the appropriate choice of $j$. This creates a new $u, v$-path $P^{\prime}$ such that $H \subseteq B \subseteq P^{\prime}$ and $\left|P \backslash P^{\prime}\right| \leq \frac{|P|-k}{k-1}$.

Before our next lemma, we observe an easy fact without proof.

Fact 2 Let $G$ be a $k$-connected graph for $k \geq 2$ and let $u$ and $v$ be two distinct vertices in $G$. Then for any $u, v$-path $P$ with $|P|<k$, there is another $u$, v-path $P^{\prime}$ with $\left|P^{\prime}\right| \geq k$ such that $P \subseteq P^{\prime}$.

Lemma 3 Let $G$ be a graph with $\kappa(G)=k$ and $\alpha(G)=\alpha$. If $u$, $v$ are two vertices in $G$, $\ell$ is an integer satisfying $0 \leq \ell \leq \alpha-k+1$, then there exists a set of $u, v$-paths $P_{0}, \ldots, P_{\ell}$ satisfying:

$$
\text { 1. } \alpha\left(G \backslash \bigcup_{i=0}^{\ell} P_{i}\right) \leq \alpha-k+1-\ell
$$


2. $\left|P_{i} \backslash \bigcup_{j=0}^{j-1} P_{j}\right| \leq \frac{\left|P_{0}\right|-k}{k-1}$ for $1 \leq i \leq \ell$

Proof: Induct on $\ell$. If $\ell=0$, Theorem 2 gives a $u$, $v$-path $P_{0}$ with $\alpha\left(G \backslash P_{0}\right) \leq \alpha-k+1$. Now suppose we have $u, v$-paths $P_{0}, \ldots, P_{\ell-1}$ satisfying Properties 1 and 2 for $\ell-1$.

Let $H=G \backslash \cup_{i=0}^{\ell-1} P_{i}$ be so that $\alpha(H) \leq \alpha-k+1-(\ell-1)$. Assume $\alpha(H) \geq 1$ since otherwise we could simply set $P_{\ell}=P_{0}$. By Lemma 2 with $P_{0}=P$ (note that Fact 2 implies we may assume $\left|P_{0}\right| \geq k$ ), there is a $u, v$-path $P^{\prime}$ such that $\left|P_{0} \backslash P^{\prime}\right| \leq \frac{\left|P_{0}\right|-k}{k-1}$ and $\alpha\left(H \backslash P^{\prime}\right) \leq \alpha(H)-1 \leq \alpha-k+1-\ell$.

Case $1\left|P^{\prime}\right| \leq\left|P_{0}\right|$

Then $\left|P^{\prime} \backslash \cup_{i=0}^{\ell-1} P_{i}\right| \leq\left|P^{\prime} \backslash P_{0}\right| \leq\left|P_{0} \backslash P^{\prime}\right| \leq \frac{\left|P_{0}\right|-k}{k-1}$, so we can set $P^{\prime}=P_{\ell}$ to satisfy the desired properties.

Case $2\left|P^{\prime}\right|>\left|P_{0}\right|$

Relabel the paths as follows: $P_{0}^{\prime}=P^{\prime}$ and $P_{i}^{\prime}=P_{i-1}$ for $1 \leq i \leq \ell$. This new labelling gives $\alpha\left(G \backslash \cup_{i=0}^{\ell} P_{i}^{\prime}\right) \leq \alpha-k+1-\ell$ so Property 1 is satisfied. For Property 2, first consider the case $i=1$. $\left|P_{i}^{\prime} \backslash P_{0}^{\prime}\right|=\left|P_{0} \backslash P^{\prime}\right| \leq \frac{\left|P^{\prime}\right|-k}{k-1}$ as desired. For $2 \leq i \leq \ell$, we have

$$
\left|P_{i}^{\prime} \backslash \bigcup_{j=0}^{i-1} P_{j}^{\prime}\right| \leq\left|P_{i-1} \backslash \bigcup_{j=0}^{i-2} P_{j}\right| \leq \frac{\left|P_{0}\right|-k}{k-1} \leq \frac{\left|P_{0}^{\prime}\right|-k}{k-1}
$$

so this labelling satisfies Properties 1 and 2, and we have our desired result.

Using these lemmas, the proof of our main result is easy.

Proof of Lemma 1: For $k=1$, the result is trivial so we will assume $k \geq 2$. When $k>\alpha$, the assertion holds by Theorem 7. Thus, we may also assume $\alpha \geq k$.

Set $\ell=\alpha-k+1$ and apply Lemma 3. By Property 1 , the set of paths $P_{0}, \ldots, P_{\ell}$ must cover all of $V(G)$. Using Property 2, this implies

$$
n=\left|P_{0}\right|+\sum_{i=1}^{\ell}\left|P_{i} \backslash \bigcup_{j=0}^{i-1} P_{j}\right| \leq\left|P_{0}\right|+(\alpha-k+1)\left(\frac{\left|P_{0}\right|-k}{k-1}\right) .
$$

Solving for $\left|P_{0}\right|$, we get get the desired result $\left|P_{0}\right| \geq \frac{(k-1)(n-k)}{\alpha}+k$.

Proof of Theorem 4: This proof is by induction on $s$. If $s=2$, the result follows immediately from Lemma 1 . Now suppose $s>3$ and consider $G \backslash v_{s}$. This graph has $\kappa\left(G \backslash v_{s}\right) \geq k-1$ and we will assume $\alpha\left(G \backslash v_{s}\right)=\alpha(G)$ (otherwise a stronger result is possible). By induction on $s$, there exists a tree $T_{s-1} \subseteq G$ with $\ell\left(T_{s-1}\right)=\left\{v_{1}, \ldots, v_{s-1}\right\}$ and 


$$
\begin{aligned}
\left|T_{s-1}\right| & \geq \min \left\{n-1, \frac{(k-s+1)(n-k)}{\alpha}+k-1, \frac{(k-s+2)(n-k-1)}{\alpha}+k\right\} \\
& \geq \min \left\{n-1, \frac{(k-s+1)(n-k)}{\alpha}+k-1\right\}
\end{aligned}
$$

as long as $n \geq 2 k+2-s-\alpha$. Otherwise, if we assume $n<2 k+2-s-\alpha$, then since $n \geq k+1$, if we let $H=G \backslash\left\{v_{3}, v_{4}, \ldots, v_{s}\right\}$, we have $\kappa(H) \geq \alpha+1$. By Theorem 7 , this means that $H$ is hamiltonian connected so we can find a path $P$ from $v_{1}$ to $v_{2}$ using all of $H$. Since $G$ is $k$-connected, each vertex $v_{i}$ for $3 \leq i \leq s$ has at least $k$ paths to $P$. Since $k \geq s$, there is an edge from each $v_{i}$ to $P \backslash\left\{v_{1}, v_{2}\right\}$, forming the desired tree of order $n$. Hence, we may suppose the above inequality holds.

In $G$, there are $k$ disjoint (except at $v_{s}$ ) paths from $v_{s}$ to $T_{s-1}$ so there is at least one such path $Q$ which avoids the set $\left\{v_{1}, \ldots, v_{s-1}\right\}$. Hence, the tree $T=T_{s-1} \cup Q$ is the desired tree with $|T| \geq\left|T_{s-1}\right|+1$.

\section{Conclusion}

The results contained in this work are all sharp by the following example. Let $C=K_{k}$ and let $H_{i}=K_{\frac{n-k}{\alpha}}$ for $1 \leq i \leq \alpha$ where we assume $\alpha$ divides $n-k$. Let $G=C+\left(\cup H_{i}\right)$ where + is the standard join operation such that $V(A+B)=V(A) \cup V(B)$ and $E(A+B)=$ $E(A) \cup E(B) \cup\{u, v: u \in A, v \in B\}$. Choose $u, v \in C$ and let $P$ be a $u, v$-path that uses all vertices of $C$ and all of $H_{1}, \ldots, H_{k-1}$. This is the longest $u, v$-path in $G$, which shows that Lemma 1 is sharp. The same example, with the inclusion of the edge $u v$ to complete a cycle, shows that Theorem 3 is sharp.

For Theorem 4 , choose $v_{1}, \ldots, v_{s}$ from $C$ to obtain the desired bound. In this situation, because these vertices must be leaves of the constructed tree, we may use the vertices of at most $k-s+1$ components $H_{i}$ in building $T$. Note also that if $s>k$, a similar result cannot hold because, if we choose all of $C$ and at least one vertex of $G \backslash C$, at least one vertex of $C$ must not be a leaf of a tree including these vertices.

The authors hope that the results contained in this work may be applied in other works. Like Theorems 3 and 4 we believe that many results will follow from this work and perhaps other proofs may be simplified through use of Lemma 1.

\section{Acknowledgement}

The authors would like to thank Garth Isaak for help on this and related projects. 


\section{References}

[1] G. Chartrand and L. Lesniak. Graphs \& Digraphs. Chapman \& Hall/CRC, Boca Raton, FL, fourth edition, 2005.

[2] V. Chvátal and P. Erdős, A note on Hamiltonian circuits, Discrete Math 2, (1972). 111-113.

[3] J.L. Fouquet and J.L. Jolivet. Problèmes combinatoires et théorie des graphes Orsay, Problèmes. 1976.

[4] S. O, D. B. West, and H. Wu. Longest cycles in $k$-connected graphs with given independence number. J. Combin. Th. (B), In Press. 